This is the final peer-reviewed accepted manuscript of:

Paolo Tortora, Marco Zannoni, Doug Hemingway, Francis Nimmo, Robert A. Jacobson, Luciano less, Marzia Parisi, Rhea gravity field and interior modeling from Cassini data analysis, Icarus, Volume 264, 2016, Pages 264-273, ISSN 0019-1035

The final published version is available online at:

https://doi.org/10.1016/j.icarus.2015.09.022

Rights / License:

The terms and conditions for the reuse of this version of the manuscript are specified in the publishing policy. For all terms of use and more information see the publisher's website.

This item was downloaded from IRIS Università di Bologna (https://cris.unibo.it/)

When citing, please refer to the published version. 


\title{
Rhea Gravity Field and Interior Modeling from Cassini Data Analysis
}

Paolo Tortora ${ }^{1 *}$, Marco Zannoni ${ }^{1}$, Doug Hemingway ${ }^{2}$, Francis Nimmo ${ }^{2}$, Robert A. Jacobson ${ }^{3}$, Luciano less ${ }^{4}$, Marzia Parisi ${ }^{4}$

${ }^{1}$ Dipartimento di Ingegneria Industriale, Università di Bologna, Forlì, Italy

${ }^{2}$ Department of Earth \& Planetary Sciences, University of California Santa Cruz, Santa Cruz, California, USA

${ }^{3}$ Jet Propulsion Laboratory, California Institute of Technology, Pasadena, California, USA

${ }^{4}$ Dipartimento di Ingegneria Meccanica e Aerospaziale, Sapienza Università di Roma, Rome, Italy

${ }^{*}$ Corresponding author.

\begin{abstract}
During its tour of the Saturn system, Cassini performed two close flybys of Rhea dedicated to gravity investigations, the first in November 2005 and the second in March 2013. This paper presents an estimation of Rhea's fully unconstrained quadrupole gravity field obtained from a joint multi-arc analysis of the two Cassini flybys.

Our best estimates of the main gravity quadrupole unnormalized coefficients are $J_{2} \times 10^{6}=946.0 \pm 13.9, C_{22}$ $\times 10^{6}=242.1 \pm 4.0$ (uncertainties are $1-\sigma$ ). Their resulting ratio is $J_{2} / C_{22}=3.91 \pm 0.10$, statistically not compatible (at a 5- $\sigma$ level) with the theoretical value of $10 / 3$, predicted for a hydrostatic satellite in slow, synchronous rotation around a planet. Therefore, it is not possible to infer the moment of inertia factor directly using the Radau-Darwin approximation.

The observed excess $J_{2}$ (gravity oblateness) was investigated using a combined analysis of gravity and topography, under different plausible geophysical assumptions. The observed gravity is consistent with that generated by the observed shape for an undifferentiated (uniform density) body. However, because the surface is more likely to be water ice, a two-layer model may be a better approximation. In this case, and assuming a mantle density of $920 \mathrm{~kg} / \mathrm{m}^{3}$, some $1-3 \mathrm{~km}$ of excess core oblateness is consistent with the observed gravity. A wide range of moments of inertia is allowed, but models with low moments of inertia (i.e., more differentiation) require greater magnitudes of excess core topography to satisfy the observations.
\end{abstract}

Keywords: Interiors; Orbit determination; Satellites, composition; Saturn, satellites.

\section{Introduction}

Discovered on December 23, 1672 by Giovanni Domenico Cassini, Rhea is the second largest moon of Saturn, with a mean radius of about $764 \mathrm{~km}$.

Before Cassini's arrival in the Saturn system, only the gravitational parameter GM was known from the analysis of Pioneer and Voyager data (Campbell \& Anderson, 1989). Using this and the estimated volume (from camera images), a bulk density of about $1200 \mathrm{~kg} / \mathrm{m}^{3}$ was derived, relatively small and compatible

\footnotetext{
${ }^{a}$ Currently with the Department of Earth and Planetary Sciences, Weizmann Institute of Science, 234 Herzl Steet, Rehovot 7610001, Israel
} 
with a mixture of about $75 \%$ by mass water ice (density $1000 \mathrm{~kg} / \mathrm{m}^{3}$ ) and $25 \%$ rock-metal (density 3000 $\left.\mathrm{kg} / \mathrm{m}^{3}\right)$.

During its mission in the Saturn system, Cassini performed four close encounters of Rhea, of which only two were devoted to gravity investigations. The first gravity flyby, referred to as R1, according to the numbering scheme used by the Cassini project, was performed on November 26, 2005, during the main mission, and the second and last gravity flyby, referred to as R4, was performed on March 9, 2013, during the Solstice mission. The main orbital and geometrical characteristics of R1 and R4 are summarized in Table 1, while Figure 1 displays the ground track of the flybys, for a time interval of about $\pm 2 \mathrm{~h}$ around the closest approach (black circles).

Radiometric data acquired during the first encounter (R1) were used to estimate the gravity field of Rhea. A first estimate (Anderson \& Schubert, 2007) was obtained under the assumption of hydrostatic equilibrium, i.e. constraining the unnormalized gravity coefficients $\mathrm{J}_{2}$ and $\mathrm{C}_{22}$ to a ratio of $10 / 3$. From this estimation, by applying the Radau-Darwin relation the authors obtained a normalized moment of inertia of about $0.3911 \pm$ 0.0045 (a value of 0.4 would imply a constant density interior). The authors concluded that the satellite's interior is a homogeneous, undifferentiated mixture of ice and rock, with possibly some compression of the ice and transition from ice I to ice II at depth.

In parallel, the radiometric data acquired during R1 were independently analyzed by the Cassini Navigation team (MacKenzie et al., 2007) and by the Cassini Radio Science team (less et al., 2007). Both analyses estimated the moon's GM and quadrupole gravity coefficients $J_{2}$ and $C_{22}$, obtaining different solutions, but consistent at the $2 \sigma$ level, as a result of different analysis approaches. The two approaches were then combined to obtain a joint "best" unconstrained estimation of the quadrupole field (Mackenzie et al., 2008). The solution obtained is not statistically compatible with hydrostatic equilibrium, hence no useful constraint on Rhea's interior structure could be imposed. Hydrostatic equilibrium was also ruled out by applying this constraint to the estimated quadrupole field coefficients, and this led to a significant degradation of the orbital fit at closest approach. To explain the non-hydrostatic ratio $J_{2} / C_{22}$, the authors theorized that a large collision occurred after the completion of the thermal evolution of the satellite, causing a redistribution of mass and a reorientation of the tidal bulge.

More recently (Anderson \& Schubert 2010) stated that the differences in the previously published gravity fields are probably caused by a mis-modeling of the non-gravitational acceleration acting on Cassini caused by anisotropic thermal emission. To avoid this issue, these authors restricted the analysis to a subset of data around the closest approach ( $\pm 2000 \mathrm{~s}$ ), where "the information from Rhea's quadrupole gravitational field is confined". They obtained a new solution in agreement with (Anderson \& Schubert, 2007), using the hypothesis of hydrostatic equilibrium. Moreover, these authors concluded that non-hydrostaticity is not supported by the data.

The different estimations of $J_{2}$ and $C_{22}$ published to date are shown in Figure 2. To resolve these discrepancies, a second and final gravity flyby was planned in Cassini's Solstice. No other flybys of Rhea are scheduled in the Cassini mission. R1 was characterized by a very low inclination, about $17^{\circ}$ at the closest approach (C/A), in order to de-correlate the estimation of $J_{2}$ and $C_{22}$, while $R 4$ was designed to be nearly polar, with a high inclination at C/A, about $106^{\circ}$. However, the C/A of R4 was about $999 \mathrm{~km}$, twice as high as R1 (about $502 \mathrm{~km}$ ), thus significantly reducing the information content about Rhea's quadrupole gravity field in this second flyby. The Sun-Earth-Probe (SEP) angle was larger than $110^{\circ}$ during both encounters, thus range-rate measurements were only slightly affected by the harmful effect of solar plasma.

This paper is organized as follows: Section 2 describes the data analysis approach for the estimation of Rhea's gravity field, along with the spacecraft dynamical model, and the data selection and calibration procedure. Section 3 provides a geophysical interpretation of the results, by means of a combined analysis of Rhea's estimated gravity and topography. Finally Section 4 summarizes our findings and conclusions. 


\section{Gravity Analysis}

\subsection{Introduction}

The determination of the gravity field of a celestial body plays a crucial role in the investigation of its internal composition, structure and evolution, because it provides one of the very few direct measurements of its internal mass distribution, even if the inversion process is not unique.

The gravity field of Rhea was precisely determined by reconstructing the trajectory of Cassini during the two close encounters of the satellite. The main observable quantity used in the gravity estimation was the spacecraft range-rate, obtained from the frequency shift due to the relativistic Doppler effect, averaged over a count time of $60 \mathrm{~s}$, of a highly stable microwave carrier transmitted from an Earth ground station to the spacecraft, that coherently retransmits the signal to Earth by means of a precise transponder. Range observables were not used in the analysis because they are of very limited use in the estimation of gravity fields.

During a close flyby, the spacecraft velocity variations caused by the main terms of the gravity field harmonic expansion are approximately (less et al., 2014a):

$$
\begin{gathered}
\Delta V(G M) \approx \frac{G M}{r V} \\
\Delta V\left(J_{2}\right) \approx \frac{G M}{r V}\left(\frac{R}{r}\right)^{2} J_{2} \\
\Delta V\left(J_{3}\right) \approx \frac{G M}{r V}\left(\frac{R}{r}\right)^{3} J_{3}
\end{gathered}
$$

where $M$ is the satellite mass, $r$ is the radial distance at closest approach, $R$ is the satellite radius, $V$ is the flyby velocity and $\mathrm{G}$ is the gravitational constant. Using the geometrical values at C/A reported in Table 1 and the gravity coefficients of (MacKenzie et al., 2008) the expected velocity variations were computed and are shown in Table 2. In this simplified analysis a conservative upper bound of $J_{3} \times 10^{6}=25$ was used, following (Mackenzie et al., 2008).

The Cassini radio science subsystem, along with the ground stations of NASA's Deep Space Network (DSN), allows a tracking accuracy in the spacecraft's velocity variations of about 0.02 to $0.09 \mathrm{~mm} / \mathrm{s}$ on a time scale of $60 \mathrm{~s}$, depending mainly on the Sun-Earth-Probe angle (less et al., 2014b).

This accuracy is well below the expected spacecraft's velocity variations induced by Rhea's $G M$ and $J_{2}$ during R1 and R4, while it is comparable to the upper bound contribution due to $\mathrm{J}_{3}$.

\subsection{Dynamical Model}

To correctly estimate the orbit of Cassini, the dynamical model implemented in the orbit determination program must take into account all non-negligible accelerations acting on the spacecraft.

The implemented dynamical model includes the point-mass relativistic gravitational acceleration exerted by all the main solar system bodies and the main satellites of Saturn. The gravitational parameters and state vectors of the planets, the Sun, the Moon and Pluto were obtained from JPL planetary ephemerides DE430 (Folkner et al., 2014). The gravitational parameters and a priori state vectors of Saturn and its main satellites were obtained from Saturn's satellites ephemerides, SAT355, provided by the Cassini Navigation Team by fitting a large number of radio, astrometric, and optical data (Jacobson et al., 2006) (Available at ftp://ssd.jpl.nasa.gov/pub/eph/satellites/). 
The dynamical model also included the gravitational acceleration due to the even zonal spherical harmonics of Saturn from $J_{2}$ up to $J_{8}$, whose updated values were provided by the satellite ephemerides, along with an updated model of Saturn's rotation. Rhea's gravity field was modeled using a degree- 2 spherical harmonics expansion, which was the minimum degree field capable of fitting the data at the noise level, without clear signatures. Given the very small orbital eccentricity (about 0.001), the periodic tidal effects of Saturn on Rhea were neglected: in this condition the tidal bulge is almost constant, and the quadrupole coefficients $J_{2}$ and $\mathrm{C}_{22}$ are a function only of the secular Love number $\mathrm{k}_{\mathrm{f}}$.

Given the very small eccentricity, Rhea is considered to be in synchronous rotation around Saturn, with the spin axis normal to the orbital plane and prime meridian always oriented toward Saturn. The rotational model adopted was based on the latest coordinate orientations adopted by the IAU (Archinal et al., 2011), with small corrections applied to obtain a better fit of Cassini and other astrometric data. The adopted Rhea rotational model is reported in Equations (4), (5), and (6).

$$
\begin{gathered}
\alpha_{0}\left(^{\circ}\right)=40.347-0.17089132 T-2.958 \sin \theta+0.076 \sin 2 \theta \\
\delta_{0}\left(^{\circ}\right)=83.550-0.01277063 \mathrm{~T}-0.332 \cos \theta+0.004 \cos 2 \theta \\
W\left(^{\circ}\right)=235.16+79.69005069 \mathrm{~d}+2.941 \sin \theta-0.077 \sin 2 \theta
\end{gathered}
$$

Where $\alpha_{0}$ and $\delta_{0}$ are the right ascension and declination of the north pole with respect to EME2000, respectively, $\mathrm{W}$ is the angle measured easterly along the body's equator between the prime meridian and the ascending line of nodes, $T$ is the time measured in Julian centuries (36525 days) past J2000, $d$ is the time measured in days past $\mathbf{J} 2000$, and $\theta$ is a nutation-precession term given by Equation (7).

$$
\theta\left(^{\circ}\right)=9.151048+1004.6342250 \mathrm{~T}
$$

A dynamically defined, perfectly synchronous, rotational model was also used, but the solution was not affected significantly.

The dynamical model also included the most important non-gravitational forces acting on Cassini during the flybys: the solar radiation pressure (SRP) and the thermal thrust. Both accelerations were described by the same models adopted by the Cassini navigation team and whose parameters were estimated using cruise and tour data.

The SRP is caused by momentum transfer between the spacecraft and the photons hitting its surface. In general a proper modeling of this acceleration is very difficult but, during tracking passes, Cassini keeps the large High Gain Antenna (HGA) pointed to the Earth, shadowing the spacecraft bus. Hence, during gravity flybys the total frontal area exposed to the Sun is nearly constant, resulting in a constant acceleration on the order of $5 \times 10^{-13} \mathrm{~km} / \mathrm{s}^{2}$ (Di Benedetto et al., 2009).

During tracking passes, SRP is essentially a function of the thermo-optical coefficients of the HGA, whose value has been computed from the readings of two temperature sensor mounted on the HGA backside, using a simple thermal model of the element (Di Benedetto et al., 2009). In order to assess the effect of an imperfect knowledge of the HGA thermo-optical coefficients their uncertainty was taken into account in the computation of the formal covariance of the solution. In particular, using a conservative uncertainty equal to $100 \%$ of the value, the solution covariance increases by a negligible amount.

The thermal thrust is caused by an anisotropic thermal emission of Cassini, and in particular caused by the thermal heat generated by the three onboard Radio-isotope Thermal Generators (RTG). Due to the spacecraft geometry, the main effect is an acceleration along the spacecraft $Z$ body-fixed axis (which is the HGA boresight axis) of about $5 \times 10^{-12} \mathrm{~km} / \mathrm{s}^{2}$ (Di Benedetto et al., 2009). 
The RTG-induced acceleration is modeled using a simple exponential model:

$$
\boldsymbol{A}(t)=\boldsymbol{A}_{\mathbf{0}} e^{-\beta\left(t-t_{0}\right)}
$$

where $\mathbf{A}(\mathrm{t})$ is the thermal acceleration vector at time $t$, in body-fixed coordinates, $\mathbf{A}_{0}$ is the spacecraft thermal acceleration at the reference epoch $t_{0}$, and $\beta$ is the time scale of the exponential law, which derives from the 87.7 -year half-life of ${ }^{238} \mathrm{Pu}$ used as nuclear fuel in the RTGs.

The magnitude of the RTG reference acceleration $\mathbf{A}_{0}$ has been estimated during the cruise phase to a $3 \%$ uncertainty for the radial component (Di Benedetto et al., 2009).

The force due to Saturn's thermal radiation incident on Cassini was neglected, because the resulting acceleration is on the order of $10^{-16} \mathrm{~km} / \mathrm{s}^{2}$, increasing up to $5 \times 10^{-15} \mathrm{~km} / \mathrm{s}^{2}$ near Saturn pericenter (Di Benedetto et al., 2009), i.e., about three orders of magnitude smaller than the RTGs acceleration.

\subsection{Data Selection and Calibration}

During the encounters, Doppler data at $X(8.4 \mathrm{GHz})$ and $\mathrm{Ka}$ band $(32.5 \mathrm{GHz})$ were acquired by the antennas of NASA's DSN at the three complexes of Goldstone, Madrid and Canberra. In addition to data obtained around the closest approach, the analysis also used data obtained up to two days before and after the closest approach, during standard navigation tracking passes. The additional data allow an improvement of the orbit determination, in particular the estimation of Rhea's ephemerides and Rhea's GM, because of the stronger constraints that are imposed on the relative trajectories between Cassini, Rhea and Saturn.

It is important to note that the closest approach of R1 was tracked only in three-way mode, meaning that the receiving and transmitting stations were different. This represents a sub-optimal condition, due to the possible delay between the reference oscillators of the two different uplink and downlink stations. During R4, the closest approach was tracked in two-way mode.

Dual frequency $X$-band uplink and Ka-band downlink (X/Ka) Doppler data were preferred to the standard $X / X$ data when available, to reduce the effects of the dispersive noise sources, mainly the solar corona and the Earth ionosphere. Two-way Doppler data were preferred to three-way data in the same band when both were available, to remove the errors due to the clock synchronization between the uplink and downlink ground stations.

When only two-way $X / X$ data and three-way $X /$ Ka data were available, the selection was made on a case by case basis.

When available, the wet path delay due to the Earth's troposphere was calibrated using measurements from advanced water vapor radiometers (Bar-Sever et al., 2007). When not available, the Earth's troposphere was calibrated using a combination of weather data and dual frequency GPS measurements.

\subsection{Estimation}

Data analysis was carried out using two different approaches, producing a multi-arc solution (SOL1) and a global solution (SOL2).

SOL1 was obtained using JPL's orbit determination program MONTE (Mission Analysis, Operations, and Navigation Toolkit Environment) and a multi-arc approach, in which radiometric data obtained during noncontiguous orbital segments, called "arcs", are jointly analyzed to produce a single solution of a set of "global" parameters, which do not vary with time.

MONTE is the new orbit determination software developed by JPL to replace the Orbit Determination Program (ODP) and it is now used for the operations of all NASA's space missions managed by JPL. MONTE 
and ODP share the same mathematical formulation (described in detail in (Moyer, 1971) and (Moyer, 2000)) which, apart from navigation, proved successful also for radio science data analysis.

The a priori values of $\mathrm{GM}, \mathrm{J}_{2}, \mathrm{C}_{22}$ and $\mathrm{S}_{22}$ were retrieved from (MacKenzie et al., 2008). The a priori values of $\mathrm{C}_{21}$ and $\mathrm{S}_{21}$ were set to zero. In order to avoid constraining the estimation, the a priori uncertainties of the $\mathrm{GM}$ and the estimated quadrupole coefficients were set to at least one order of magnitude larger than their formal uncertainty. No hydrostatic equilibrium constraint between $J_{2}$ and $C_{22}$ was imposed.

To properly fit the radiometric data, an updated state vector of Rhea at a reference epoch was estimated, and the ephemerides of all main Saturn satellites were updated by numerically integrating the equations of motion, in order to keep constant the mass and the trajectory of the system's center of mass. The a priori values for the Rhea state were retrieved from the Saturn satellite ephemerides SAT355.

For each arc, a separate initial condition for the Cassini state vector was estimated. The a priori values were obtained from the reconstructed trajectory provided by the Cassini Navigation Team (The updated Cassini trajectory Spice kernels are available at ftp://naif.jpl.nasa.gov/.).

The residuals were weighted on a pass-by-pass basis using their own RMS value.

Solution SOL2 was made using JPL's original orbit determination program, the ODP. Moreover, it was obtained as part of the ongoing global analysis that seeks to determine the satellite ephemerides, Saturn's rotational model, and the gravitational parameters and gravity fields of Saturn and it major satellites. The global analysis, described in detail in (Jacobson et al. 2006), utilizes an extensive data set that includes: Earth-based and Hubble Space Telescope astrometry, satellite mutual events, Saturn ring occultations, imaging from the Voyager and Cassini spacecraft, and radiometric tracking of Pioneer 11, Voyager, and Cassini.

As in the SOL1 solution, the Doppler data that are sensitive to the gravity parameters are weighted pass-bypass at the RMS of their residuals. However, the data are pre-processed to remove the correlation between data points caused by scintillation in the solar plasma, assumed to be the dominant noise source and having a spectrum that follows the Kolmogorov power law.

\subsection{Results}

Figure 3 and Figure 4 plot the post-fit residuals of the tracking passes around the closest approaches of R1 and R4. The residuals do not show any evident signature around the closest approach, the mean is approximately zero and the RMS are about $76 \mu \mathrm{m} / \mathrm{s}$ for R1 and about $17 \mu \mathrm{m} / \mathrm{s}$ for R4.

Figure 5 shows the different estimations of Rhea's unnormalized $J_{2}$ and $C_{22}$ in the $J_{2}-C_{22}$ plane, along with their 1- $\sigma$ error ellipses, compared to the results published in (MacKenzie et al., 2008) and (Anderson \& Schubert, 2010). As a reference, Table 3 collects all the estimated gravity coefficients for all the different solutions displayed in Figure 5.

Although obtained with two independent analyses and following different approaches, SOL1 and SOL2 are statistically compatible: the difference between the estimated values of $J_{2}$ and $S_{22}$ is less than 1- $\sigma$, while the difference in the estimates of $C_{22}$ is within 3- $\sigma$. Moreover, both solutions are statistically compatible with (Mackenzie et al., 2008). This provides strong evidence of the reliability and robustness of the solution. However, the discrepancy with (Anderson \& Schubert, 2010) is statistically significant, being larger than 3- $\sigma$ and 5- $\sigma$ for $J_{2}$ and $C_{22}$, respectively. Recall, however, that the (Anderson \& Schubert, 2010) solution was obtained by constraining the hydrostatic ratio $J_{2} / C_{22}$ to $10 / 3$. Applying the same condition, our solution becomes fully compatible with (Anderson \& Schubert, 2010), but the residuals show a large signature at the closest approach of R1, in a similar way as (MacKenzie et al., 2008). Moreover, considering only a time interval of \pm 15 minutes around $C / A$, where almost the entire quadrupole field signal on the range-rate is concentrated, the RMS of the residuals increases by about $56 \%$, from $64 \mu \mathrm{m} / \mathrm{s}$ to $100 \mu \mathrm{m} / \mathrm{s}$, a strong indication of a wrong dynamical model. Given the larger altitude at the closest approach of R4, applying the 
hydrostatic constraint the residuals of that pass do not show any evident signature, while their RMS increases only slightly, from $17 \mu \mathrm{m} / \mathrm{s}$ to $18 \mu \mathrm{m} / \mathrm{s}$.

From MacCullagh's theorem the quadrupole gravity coefficients are related to the body's inertia tensor. In particular, $\mathrm{J}_{2}$ and $\mathrm{C}_{22}$ are a function of the differences between the diagonal terms, while $\mathrm{C}_{21}, \mathrm{~S}_{21}$, and $\mathrm{S}_{22}$ are a function of the off-diagonal terms, which are null in a frame of principal axes of inertia.

Under the assumption of small rotations, $\mathrm{C}_{21}$ and $\mathrm{S}_{21}$ are related to a misalignment between the assumed spin axis and the real maximum inertia axis, while $S_{22}$ is related to a misalignment between the adopted reference frame and the principal axes frame around the spin axis.

The estimated values of $C_{21}$ and $S_{21}$ are null within 2- $\sigma$, as expected, while $S_{22}$ is slightly larger, being about 3- $\sigma$ away from zero. However, this proved to be due to the particular choice of the adopted Rhea-fixed reference frame, as shown in the following. Indeed, Rhea's prime meridian used in the model and the Saturn-pointing direction were found to be misaligned by about $3^{\circ}$, probably because the former was retrieved using different Satellite ephemerides and Rhea's orbit was updated as part of the fitting process. Instead, using a perfectly synchronous frame, dynamically defined using Rhea's updated orbit, $S_{22}$ decreases becoming null within $2-\sigma$, while $C_{22}$ increases in a statistically non-significant way. Therefore, there is a good alignment within uncertainty between the principal inertia axis of Rhea and a synchronous rotational state. Rhea's quadrupole is dominated by $\mathrm{J}_{2}$ and $\mathrm{C}_{22}$, as expected by a satellite in synchronous rotation around its planet. However, the ratio $J_{2} / C_{22}$ is $3.91 \pm 0.10$ for SOL1 and $4.22 \pm 0.19$ for SOL2, which are equivalent to each other at the 2- $\sigma$ level and more than 5- $\sigma$ away from the hydrostatic value of 10/3. Therefore Rhea's gravity field is significantly non-hydrostatic, meaning that the moment of inertia cannot be inferred directly from either $\mathrm{J}_{2}$ or $\mathrm{C}_{22}$ using the Radau-Darwin approximation. Estimating the moment of inertia directly from the $\mathrm{J}_{2}$ or $\mathrm{C}_{22}$ coefficients of SOL1 yields incompatible and unrealistic values of about 0.40 and 0.38 , respectively.

As pointed out by (Anderson \& Schubert, 2010), errors in the thermal acceleration model may produce biases in the estimation of the gravity coefficients. In particular, a relatively high correlation (about 0.71 ) between Rhea's $J_{2}$ and the radial component of the thermal acceleration was found. To take into account this possible error source, an updated value of the reference thermal acceleration vector was estimated, using a $5 \%$ a priori uncertainty for the radial component, and a $10 \%$ a priori uncertainty for the non-radial components.

The estimated values of the thermal acceleration are compatible within 1- $\sigma$ with the a priori values, which were adopted by the Cassini navigation team. The formal uncertainty in the radial component is $3 \%$, while the uncertainties in the other components are equal to the a priori value of $10 \%$. Therefore, using data collected during Rhea's flybys, only the knowledge of the radial component of the thermal acceleration could be improved, because Doppler measurements are directly sensitive only to velocity variations along the line of sight.

To test the stability of the solution the a priori uncertainties of the thermal accelerations were increased to $100 \%$ of their a priori value, which can be considered a conservative upper bound. Rhea's $J_{2}$ and $C_{22}$ change by less than $2-\sigma$, their formal uncertainty increase by about $25 \%$ and $7 \%$, respectively, and the ratio $J_{2} / C_{22}$ becomes $3.85 \pm 0.11$, still $4.8-\sigma$ away from the hydrostatic value. Therefore, we conclude that even a modelization error in the thermal acceleration of $100 \%$ is not sufficient to absorb the excess of the $J_{2} / C_{22}$ ratio with respect to the hydrostatic value.

\section{Interpretation}

There are a number of ways to explain the excess oblateness (greater than hydrostatic $J_{2} / C_{22}$ ratio) in Rhea's gravity field that are consistent with the observed shape, which has been determined via analysis of limb profiles (e.g., Thomas et al., 2007; Thomas et al. 2010; Nimmo et al., 2011). Incorporating the latest 
available data (Thomas, pers. comm., 2014), we adopt for this analysis a best-fitting triaxial ellipsoid with semi-axes:

$$
\begin{aligned}
& a=765.68 \pm 0.25 \mathrm{~km} \\
& b=763.60 \pm 0.25 \mathrm{~km} \\
& c=762.86 \pm 0.15 \mathrm{~km}
\end{aligned}
$$

from which we obtain the unnormalized degree-2 shape coefficients: $J_{2}=1190 \pm 260 \mathrm{~m}$ and $C_{22}=350 \pm 120 \mathrm{~m}$ (uncertainties are all one-sigma) (blue square and crosshairs in Figure 6). The large uncertainties result from Rhea's relatively small tidal/rotational distortion and rough topography. The shape is not as well determined as the gravity; for instance, the topography coefficients are consistent (within error) with a hydrostatic body, while the gravity coefficients are not.

In the analysis that follows we will assume that the degree-2 coefficients provide a record of the time the shape and gravity were "frozen in". We do not consider the possibility that these coefficients were modified by later processes, such as the formation of impact basins. Most such basins are sufficiently small that they will not have an appreciable degree-2 signature. Tirawa represents a possible exception, but we do not consider this issue further here.

We first take the simplest case of a homogeneous, uniform density Rhea. For a body of uniform density, it can be shown (Jeffreys, 1976; Hemingway et al., 2013; less et al., 2014a) that the shape required to explain the observed gravity is given by

$$
H_{l m}=\frac{(2 l+1) R}{3} G_{l m}
$$

where $R$ is Rhea's mean radius, and $G_{l m}$ and $H_{l m}$ are spherical harmonic coefficients of degree $I$ and order $m$, representing the dimensionless gravitational potential and the topography, respectively. The green circle and crosshairs in Figure 6 illustrate the topography coefficients obtained from equation (10), representing the shape required for a homogeneous Rhea to match the observed gravity field (for clarity, we use only SOL1 for the remainder of this analysis; results are not significantly different when we use SOL2 instead). The large uncertainties in the shape allow for the possibility of a homogeneous Rhea (we will discuss this issue further below). However, because the surface is likely primarily water ice (Clark et al., 1981; Stephan et al., 2012) we next consider a two-layer model consisting of an $\mathrm{H}_{2} \mathrm{O}$ mantle overlying a denser core. Of course, as soon as the assumption of homogeneity is abandoned, the problem becomes non-unique. Nonetheless, below we focus on the two-layer model because it is relatively generic, and also consistent with the inferred structures of differentiated icy satellites such as Enceladus (less et al., 2014a).

In general, and setting aside the point mass term, the dimensionless gravitational potential coefficients for a two-layer body, referenced to radius $R$, assuming constant density in each layer, is given by a generalization of equation (10) (e.g., Lefevre et al., 2014):

$$
G_{l m}(R)=\frac{3}{(2 l+1) R \bar{\rho}}\left[\rho_{m} H_{l m}^{S}+\Delta \rho H_{l m}^{c}\left(\frac{R_{c}}{R}\right)^{l+2}\right]
$$

where the superscripts on the $H_{l m}$ terms refer to the surface (s) and core (c) topographies, $\bar{\rho}$ is Rhea's bulk density $\left(1236 \mathrm{~kg} / \mathrm{m}^{3}\right), \rho_{m}$ is the mantle density, which we take to be $920 \mathrm{~kg} / \mathrm{m}^{3}, \Delta \rho\left(=\rho_{c}-\rho_{m}\right)$ is the density contrast at the core-mantle-boundary, and $R_{c}$ is the mean core radius. Hence, the observed gravity $\left(G_{l m}\right)$ can be accommodated by a combination of core and surface topographies.

If we assume zero core topography (i.e., a spherical core), then significant surface topography (mass anomalies at the top of the $\mathrm{H}_{2} \mathrm{O}$ mantle) would be required to account for the $\mathrm{J}_{2}$ and $\mathrm{C}_{22}$ terms in the 
observed gravity field. Figure 6 illustrates that this scenario (red triangle and crosshairs) is not compatible with the observed shape at the one-sigma level, although it remains compatible at the two-sigma level. The case of a spherical core is not meant to be realistic but rather to serve as a point of reference for subsequent discussion.

The addition of topography at the core mantle boundary would contribute additional mass anomalies that, if placed in-phase, could reduce the need for large surface topography to account for the observed gravity field. We start by assuming a weak (fluid) core and relax this assumption below. Taking a theory-of-figures approach (Murray \& Dermott, 1999; Tricarico, 2014), we compute the expected hydrostatic core topography $\left(H_{l m}^{c}\right)$ and use equation (11) to compute the surface topography $\left(H_{l m}^{S}\right)$ required to account for the remainder of the observed gravity $\left(G_{l m}\right)$. We carry out this calculation for various mantle thicknesses ranging from $5 \mathrm{~km}$ (representing the maximum likely moment of inertia) to $300 \mathrm{~km}$ (corresponding to significant differentiation, with a normalized moment of inertia of $\sim 0.335$ ). The resulting surface topographies (Figure 6) are consistent with the observed topography at the one-sigma level, provided the mantle thickness is less than about $200 \mathrm{~km}$.

The presence of deep impact basins such as Tirawa ( $5 \mathrm{~km}$ in depth) suggests that the present day icy surface is rigid enough to support considerable topography on spatial scales of up to a few hundred km. Support for degree-2 topography, however, may be more limited, especially if the degree-2 shape and gravity field were established early, when Rhea's heat flux was still high (White et al., 2013). In that case, it may be more appropriate to assume that the $J_{2}\left(=-C_{20}\right)$ and $C_{22}$ surface topography is relaxed and therefore conforms closely to the geoid (equipotential surface at reference radius $R$ ), given by

$$
\begin{aligned}
& H_{20}^{\text {geoid }}=R\left(G_{20}-\frac{5}{6} q\right) \\
& H_{22}^{\text {geoid }}=R\left(G_{22}+\frac{1}{4} q\right)
\end{aligned}
$$

where $q=\left(R^{3} \omega^{2}\right) /(G M)$ is the ratio of centrifugal to gravitational acceleration, with $\omega$ being Rhea's rate of rotation (currently once every $\sim 4.5$ days).

In this scenario of the surface conforming to the geoid, an irregular (non-hydrostatic) core shape is required to account for the observed gravity. We can use equation (11) to compute the required core shape $\left(H_{l m}^{c}\right)$, setting the degree-2 surface topography $\left(H_{l m}^{S}\right)$ to match the present day geoid obtained from equation (12). For degree 2, the efficacy of adding core topography in balancing equation (11) depends linearly on core radius (because $\Delta \rho$ is proportional to $R_{c}{ }^{-3}$ ). That is, the gravity observations can be most efficiently accommodated by core topography when the $\mathrm{H}_{2} \mathrm{O}$ mantle is thin. With larger mantle thicknesses, the coremantle-boundary is deeper and so larger amplitudes of core topography are required to give rise to the same gravity signal at the surface. Figure 7 shows, for various mean mantle thicknesses, the core topography required to accommodate the observed gravity, assuming the surface topography conforms to the geoid. The magnitude of the required core topography is not much greater than what is expected for a hydrostatic core. Even in the case of a thick $(300 \mathrm{~km})$ mantle, the required excess core topography (i.e., beyond the hydrostatic expectation) is less than $2 \mathrm{~km}$ (on a core of $\sim 460 \mathrm{~km}$ radius). For comparison, Vesta's longest and shortest dimensions are roughly $573 \mathrm{~km}$ and $446 \mathrm{~km}$, suggesting that much larger core topography is likely to be easily supportable. Thomas et al. (2007) and McKinnon (2013) have likewise argued that a rigid, distorted core is plausible for Enceladus and Tajeddine et al. (2014) have discussed a similar scenario regarding Mimas.

\section{Conclusions}

The main message of this paper is that the addition of a second gravity flyby has allowed independent estimates of Rhea's $J_{2}$ and $C_{22}$ without having to make a priori assumptions. The ratio of the degree- 2 gravity coefficients $\left(\mathrm{J}_{2} / \mathrm{C}_{22}=3.91 \pm 0.10\right)$ indicates a statistically significant departure from the expected hydrostatic ratio of $10 / 3$. As a consequence, the moment of inertia cannot be inferred directly using the Radau-Darwin relation. 
As a point of comparison, the $J_{2} / C_{22}$ ratio for Enceladus was recently determined by (less et al., 2014a) to be $3.51 \pm 0.02$, also significantly in excess of the hydrostatic ratio (note that whereas $10 / 3$ is a good approximation for Rhea, the appropriate hydrostatic ratio to assume for Enceladus is closer to 3.24, due to its rapid rate of rotation (McKinnon, 2015; Tricarico, 2014)). The presence of significant non-hydrostatic topography at Enceladus suggested a differentiated interior with an isostatically compensated ice shell (less et al., 2014a). In contrast, Rhea's large non-hydrostatic gravity and small topography are more suggestive of internal mass anomalies that have little or no surface expression, as might be expected for a body with an irregular core surrounded by a weak, relaxed mantle (e.g., McKinnon, 2013).

We investigated a number of internal structure models, none of which can be strictly excluded due to the large uncertainties in the observed shape and the inherent non-uniqueness of the problem. The observed gravity and topography are consistent with Rhea being of uniform density (undifferentiated). However, spectroscopic observations suggest that the surface is more likely to be uncontaminated water ice down to at least a few kilometers, leading us to prefer a two-layer model. Two-layer models, with water ice mantles overlying denser cores, can accommodate the gravity observations with modest amounts of topography at the surface and/or at the core mantle boundary. However, if the current degree- 2 shape was established early, during an epoch of high heat flux, then the degree-2 water ice surface topography may conform closely with the geoid, in which case the majority of the excess $J_{2}$ gravity must be accommodated by an irregular core (McKinnon, 2013). Low moment of inertia models (corresponding to highly differentiated interiors) are permitted and require only a modestly oblate core (less than $2 \mathrm{~km}$ of excess core topography) and/or hundreds of meters of excess surface topography.

It is evident that the absence of hydrostatic equilibrium renders any conclusions non-unique, and this problem is compounded by the relatively poor shape determination. How might this situation be improved? Theoretical arguments of whether Rhea should be differentiated are not useful; even much larger bodies like Titan could theoretically remain undifferentiated if they accreted sufficiently gently (Barr et al. 2010). Potentially more promising are observational ways of determining the normalized moment of inertia $\left(C / M R^{2}\right)$ of Rhea.

One possibility is to measure Rhea's obliquity. As argued by Bills and Nimmo (2008), given $\mathrm{J}_{2}$ and $\mathrm{C}_{22}$, a measurement of the obliquity is then sufficient to determine the normalized moment of inertia of a satellite, assuming that it is in a Cassini state. Chen et al. (2014) calculated a predicted obliquity for Rhea of $0.03^{\circ}$ (assuming hydrostatic equilibrium). This translates into a surface distance of $0.4 \mathrm{~km}$, which would be hard to detect with optical images, but perhaps not entirely out of the question.

Since $C_{22}$ is known, one could also measure the longitudinal libration amplitude to determine the moment of inertia, as was done at Mimas by Tajeddine et al. (2014). Unfortunately, Rhea's comparative lack of tidal distortion and its extremely low eccentricity $(\mathrm{e}=0.001)$ conspire to produce a very small libration amplitude. For a normalized moment of inertia of 0.35 , the amplitude is $12.6 \mathrm{~m}$ ( $c f$. Comstock and Bills 2003). Optical images would not be sufficient to resolve such a small displacement, although radar measurements might.

In spite of the improved determination of Rhea's gravity field, the significant uncertainties in shape prevent us from drawing strong conclusions about the interior structure. In particular, neither undifferentiated nor fully-differentiated structures can be excluded. Without additional observational constraints, which may be difficult to obtain, further insights into Rhea's internal structure may remain elusive.

\section{Acknowledgements}

The authors are grateful to John W. Armstrong and Sami W. Asmar of the Cassini Radio Science Team, for the useful discussions and suggestions regarding the procedures for Cassini data analysis. Thanks to Peter Thomas for his helpful feedback and for providing the updated solution for Rhea topography used in this work. P.T., M.Z., L.I., and M.P. acknowledge support from the Italian Space Agency. D.H., and F.N. are grateful to NASA for support through the Cassini Project. The work of R.A.J. was carried out at the Jet 
Propulsion Laboratory, California Institute of Technology, under a contract with NASA. The Doppler data and ancillary information used in this analysis are archived in NASA's Planetary Data System. We thank the Cassini Project, A. Anabtawi and the JPL Radio Science Systems Group, the NASA/JPL Deep Space Network and their operations personnel involved in the acquisition of Doppler data analyzed here.

\section{References}

Anderson, J. D., and Schubert, G., "Saturn's satellite Rhea is a homogeneous mix of rock and ice, "Geophysical Research Letters, vol. 34, Jan. 2007, pp. 4-7.

Anderson, J. D., and Schubert, G., "Rhea's gravitational field and interior structure inferred from archival data files of the 2005 Cassini flyby," Physics of the Earth and Planetary Interiors, vol. 178, Feb. 2010, pp. 176-182.

Archinal, B. A., A'hearn, M. F., Bowell, E., Conrad, A., Consolmagno, G. J., Courtin, R., Fukushima, T., Hestroffer, D., Hilton, J. L., Krasinsky, G. A., Neumann, G., Oberst, J., Seidelmann, P. K., Stooke, P., Tholen, D. J., Thomas, P. C., and Williams, I. P., "Report of the IAU working group on cartographic coordinates and rotational elements: 2009," Celestial Mechanics and Dynamical Astronomy, vol. 109, 2011, pp. 101-135.

Bar-Sever, Y. E., Jacobs, C. S., Keihm, S., Lanyi, G. E., Naudet, C. J., Rosenberger, H. W., Runge, T. F., Tanner, A. B., and Vigue-Rodi, Y., "Atmospheric Media Calibration for the Deep Space Network," Proceedings of the IEEE, vol. 95, Nov. 2007, pp. 2180-2192.

Bills, B.G., Nimmo, F., 2008. Forced obliquity and moments of inertia of Titan. Icarus 196, 293-297. doi: 10.1016/j.icarus.2008.03.002

Campbell, J. K., Anderson, J. D., "Gravity field of the saturnian system from Pioneer and Voyager tracking data," Astronomical Journal, vol. 97, 1989, pp. 1485-1495.

Chen, E. M. A., F. Nimmo, and G. A. Glatzmaier. "Tidal heating in icy satellite oceans." Icarus 229 (2014): 1130. doi: 10.1016/j.icarus.2013.10.024.

Clark, Roger N., and P. D. Owensby. "The infrared spectrum of Rhea." Icarus 46.3 (1981): 354-360.

Comstock, Robert L., and Bruce G. Bills. "A solar system survey of forced librations in longitude." Journal of Geophysical Research: Planets (1991-2012) 108.E9 (2003). doi: 10.1029/2003JE002100.

Di Benedetto, M., less, L., and Roth, D. C., "The Non-Gravitational Accelerations of the Cassini Spacecraft," Proceedings of 21st International Symposium on Space Flight Dynamics, Tolouse, 2009.

Folkner, W. M., Williams, J. G., Boggs, D. H., Park, R. S., and Kuchynka, P., "The Planetary and Lunar Ephemerides DE430 and DE431," The Interplanetary Network Progress Report, 2014, pp. 1-81.

Hemingway, D., Nimmo, F., Zebker, H., and less, L., "A rigid and weathered ice shell on Titan," Nature, 500(7464):550-552, August 2013. ISSN 0028-0836. doi: 10.1038/nature12400.

less, L., Rappaport, N. J., Tortora, P., Lunine, J. I., Armstrong, J. W., Asmar, S. W., Somenzi, L., and Zingoni, F., "Gravity field and interior of Rhea from Cassini data analysis," Icarus, vol. 190, Oct. 2007, pp. 585-593.

L. less, D. J. Stevenson, M. Parisi, D. Hemingway, R. A. Jacobson, J. I. Lunine, F. Nimmo, J. W. Armstrong, S. W. Asmar, M. Ducci, and P. Tortora. The Gravity Field and Interior Structure of Enceladus. Science, 344(6179):78-80, 2014. doi: 10.1126/science.1250551. 
less, L., Di Benedetto, M., James, N., Mercolino, M., Simone, L., Tortora, P., 2014. Astra: Interdisciplinary study on enhancement of the end-to-end accuracy for spacecraft tracking techniques. Acta Astronaut. 94, 699-707. doi:10.1016/j.actaastro.2013.06.011

Jacobson, R. A., Antreasian, P. G., Bordi, J. J., Criddle, K. E., Ionasescu, R., Jones, J. B., Mackenzie, R. A., Meek, M. C., Parcher, D. W., Pelletier, F. J., Owen, W. M., Roth, D. C., Roundhill, I. M., and Stauch, J. R., "The Gravity Field of the Saturnian System from Satellite Observations and Spacecraft Tracking Data," The Astronomical Journal, vol. 132, Jan. 2006, pp. 2520-2526.

Harold Jeffreys. The Earth: its origin, history, and physical constitution. Cambridge University Press, New York, 6th edition, 1976.

Axel Lefevre, Gabriel Tobie, Gael Choblet, and Ondrej Cadek. Structure and dynamics of Titan's outer icy shell constrained from Cassini data. Icarus, 237: 16-28, July 2014. ISSN 00191035. doi: 10.1016/j.icarus.2014.04.006.

Mackenzie, R. A., Antreasian, P. G., Bordi, J. J., Criddle, K. E., Ionasescu, R., Jacobson, R. A., Jones, J. B., Parcher, D. W., Pelletier, F. J., Roth, D. C., and Stauch, J. R., "A Determination of Rhea's Gravity Field From Cassini Navigation Analysis," Proceedings of the AAS/AIAA 17th Space Flight Mechanics Meetings, 2007.

Mackenzie, R. A., less, L., Tortora, P., and Rappaport, N. J., "A non-hydrostatic Rhea," Geophysical Research Letters, vol. 35, Mar. 2008, pp. 1-5.

McKinnon, W. B., "The shape of Enceladus as explained by an irregular core: Implications for gravity, libration, and survival of its subsurface ocean," Journal of Geophysical Research: Planets, Vol. 118, pp. 1775-1788, 2013. doi:10.1002/jgre.20122.

McKinnon, W. B., "Effect of Enceladus's rapid synchronous spin on interpretation of Cassini gravity," Geophysical Research Letters, Vol. 42, 2015. doi:10.1002/2015GL063384.

Moyer, T. D., "Mathematical formulation of the Double-Precision Orbit Determination Program (DPODP)," Technical Report 32-1527, Jet Propulsion Laboratory, Pasadena (CA), 1971.

Moyer, T. D., "Formulation for Observed and Computed Values of Deep Space Network Data Types for Navigation", Wiley, Hoboken (NJ), 2000. doi:10.1002/0471728470

F. Nimmo, B. G. Bills, and P. C. Thomas, "Geophysical implications of the long-wavelength topography of the Saturnian satellites," J. Geophys. Res., 116 (E11):E11001, November 2011. ISSN 0148-0227. doi: 10.1029/2011JE003835.

Stephan, Katrin, et al. "The Saturnian satellite Rhea as seen by Cassini VIMS." Planetary and Space Science 61.1 (2012): 142-160. doi: 10.1016/j.pss.2011.07.019.

R. Tajeddine, N Rambaux, V Lainey, S Charnoz, A Richard, A Rivoldini, and B Noyelles. Constraints on Mimas' interior from Cassini ISS libration measurements. Science 346(6207):322-324, 2014.

P. Thomas, J. Burns, P. Helfenstein, S. Squyres, J. Veverka, C. Porco, E. Turtle, A. Mcewen, T. Denk, and B. Giese, "Shapes of the saturnian icy satellites and their significance," Icarus, 190(2):573-584, October 2007. ISSN 00191035. doi: 10.1016/j.icarus.2007.03.012.

Tricarico, Pasquale. "Multi-layer Hydrostatic Equilibrium of Planets and Synchronous Moons: Theory and Application to Ceres and to Solar System Moons." The Astrophysical Journal 782 (2014): 99. doi: 10.1088/0004-637X/782/2/99.

White, Oliver L., Paul M. Schenk, and Andrew J. Dombard. "Impact basin relaxation on Rhea and lapetus and relation to past heat flow." Icarus 223.2 (2013): 699-709. doi: 10.1016/j.icarus.2013.01.013. 



\section{Figures}

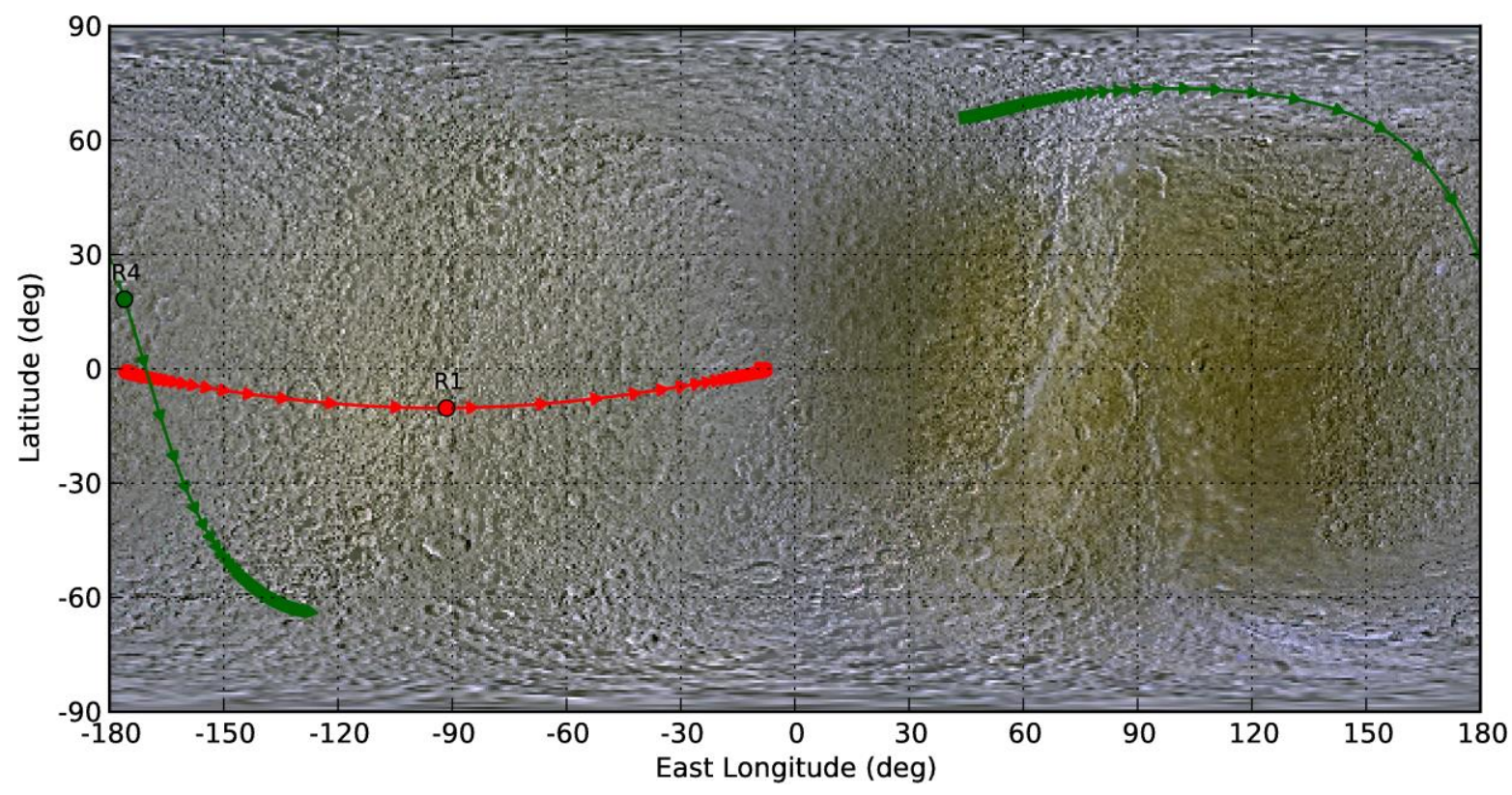

Figure 1 Cassini ground track on Rhea during R1 and R4, considering a time interval of $\pm 2 \mathrm{~h}$ around the closest approach.

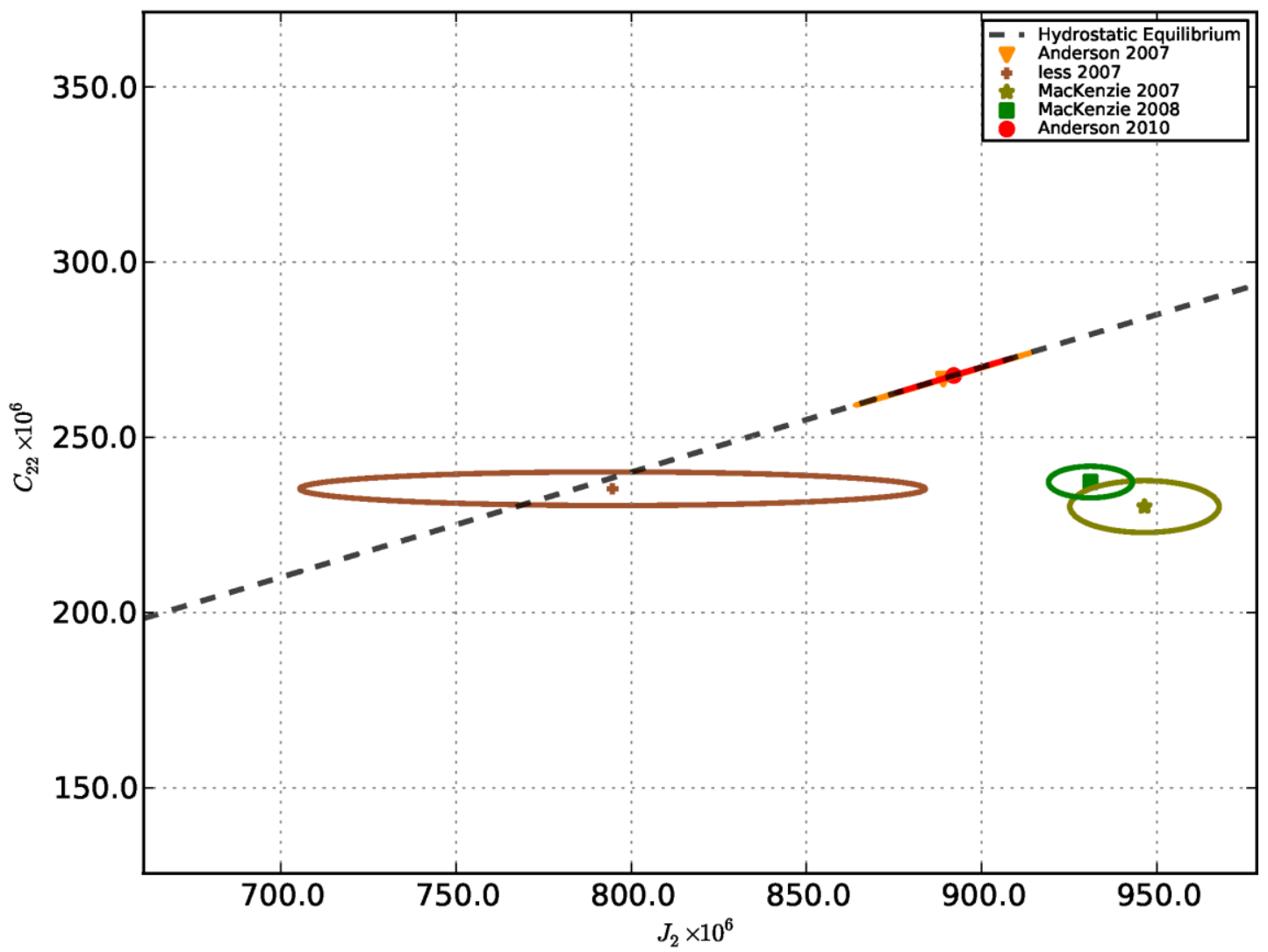

Figure 2 Previously published values of Rhea's quadrupole coefficients $\mathrm{J}_{2}$ and $\mathrm{C}_{22}$. 


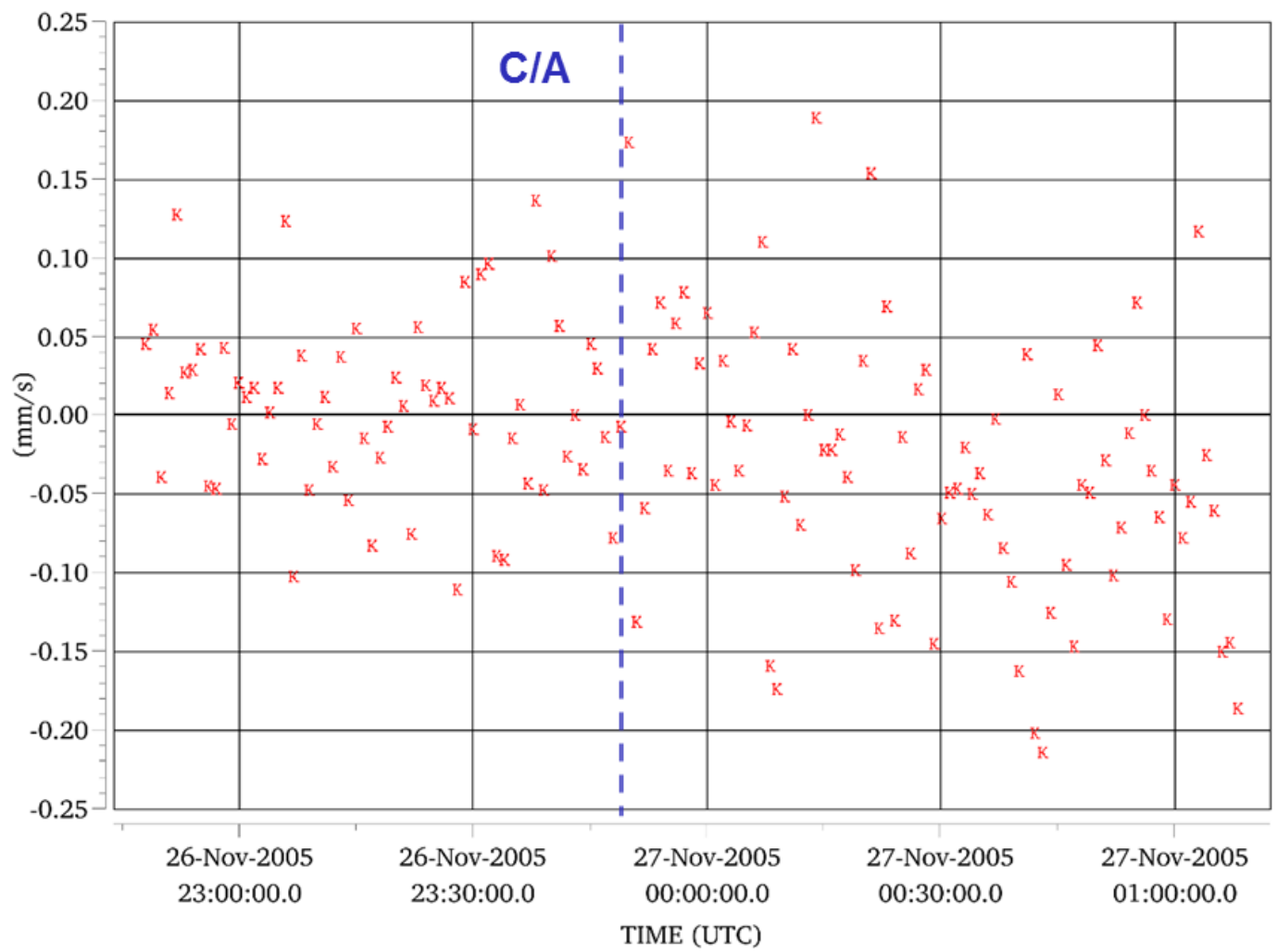

Figure 3 Range-rate residuals around the closest approach of R1 (dashed vertical line), in mm/s. The RMS is about $76 \mu \mathrm{m} / \mathrm{s}$.

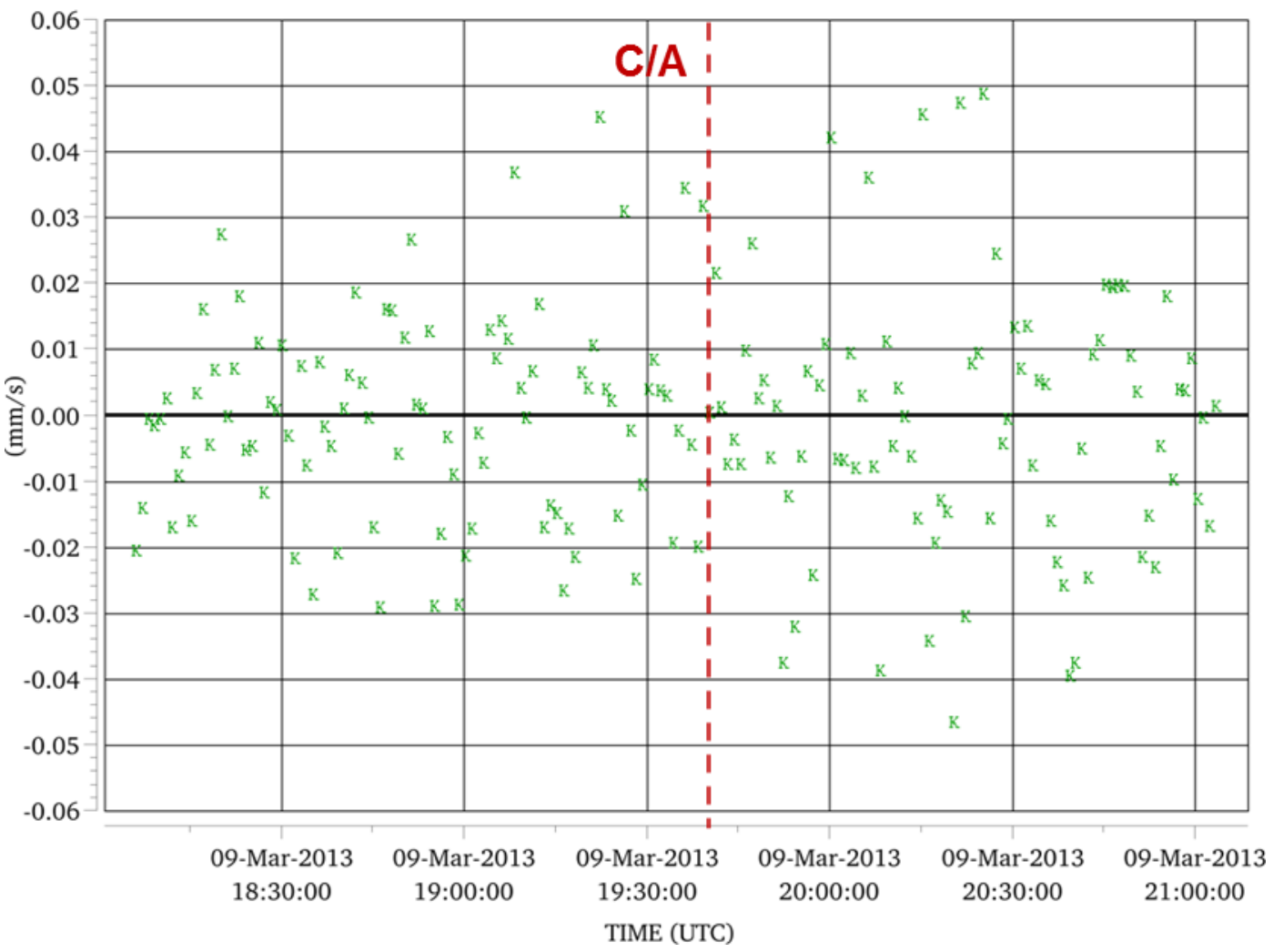

Figure 4 Range-rate residuals around the closest approach of R4 (dashed vertical line). The RMS is about $17 \mu \mathrm{m} / \mathrm{s}$. 


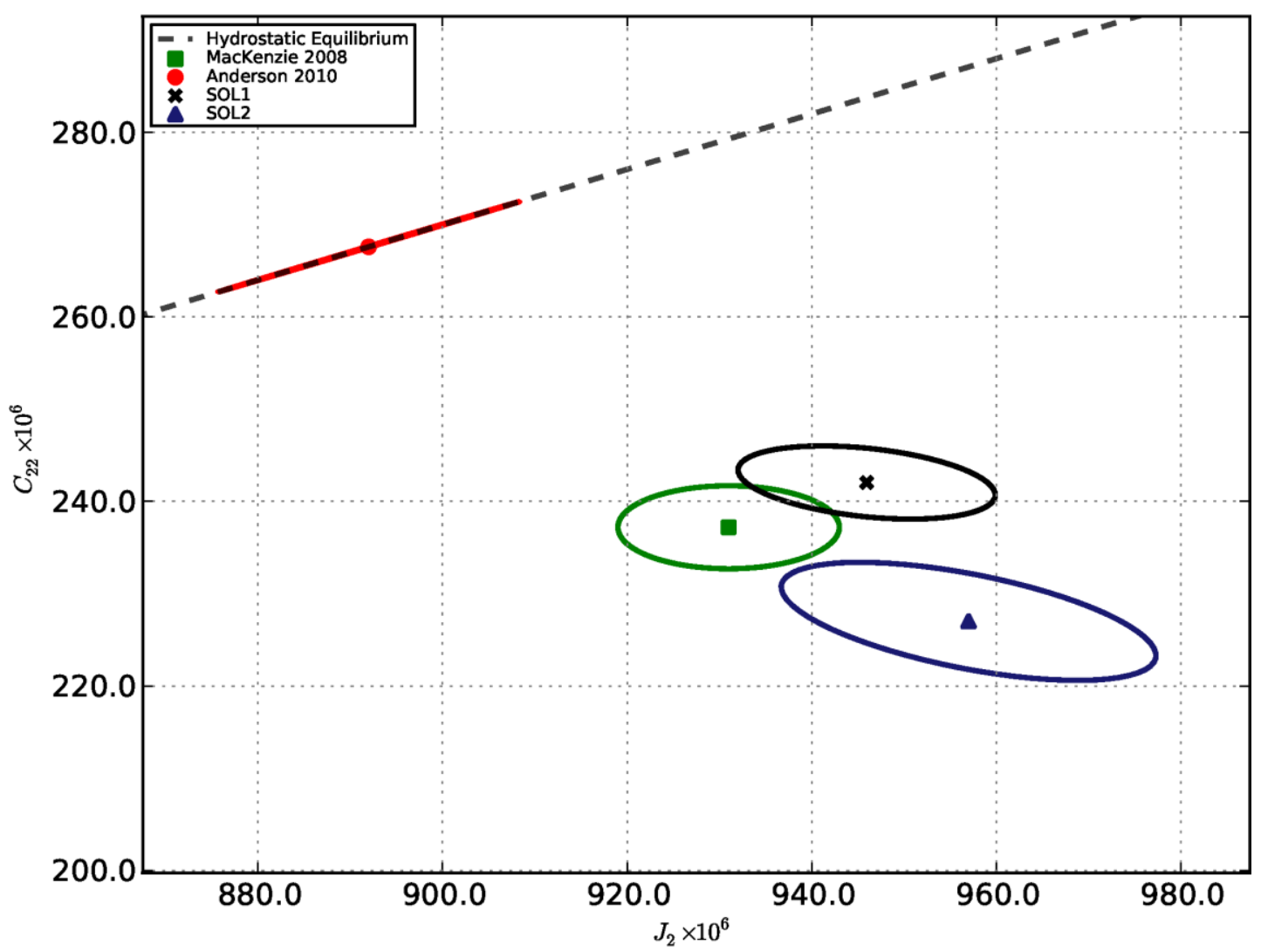

Figure 5 Estimated $J_{2}$ versus $C_{22}$ and formal 1- $\sigma$ uncertainty ellipses for the different approaches described in this paper. The most recent published values are also shown as reference. (Anderson \& Schubert, 2010) solution was obtained by constraining the $\mathrm{J}_{2} / \mathrm{C}_{22}$ ratio to the hydrostatic value $10 / 3$.

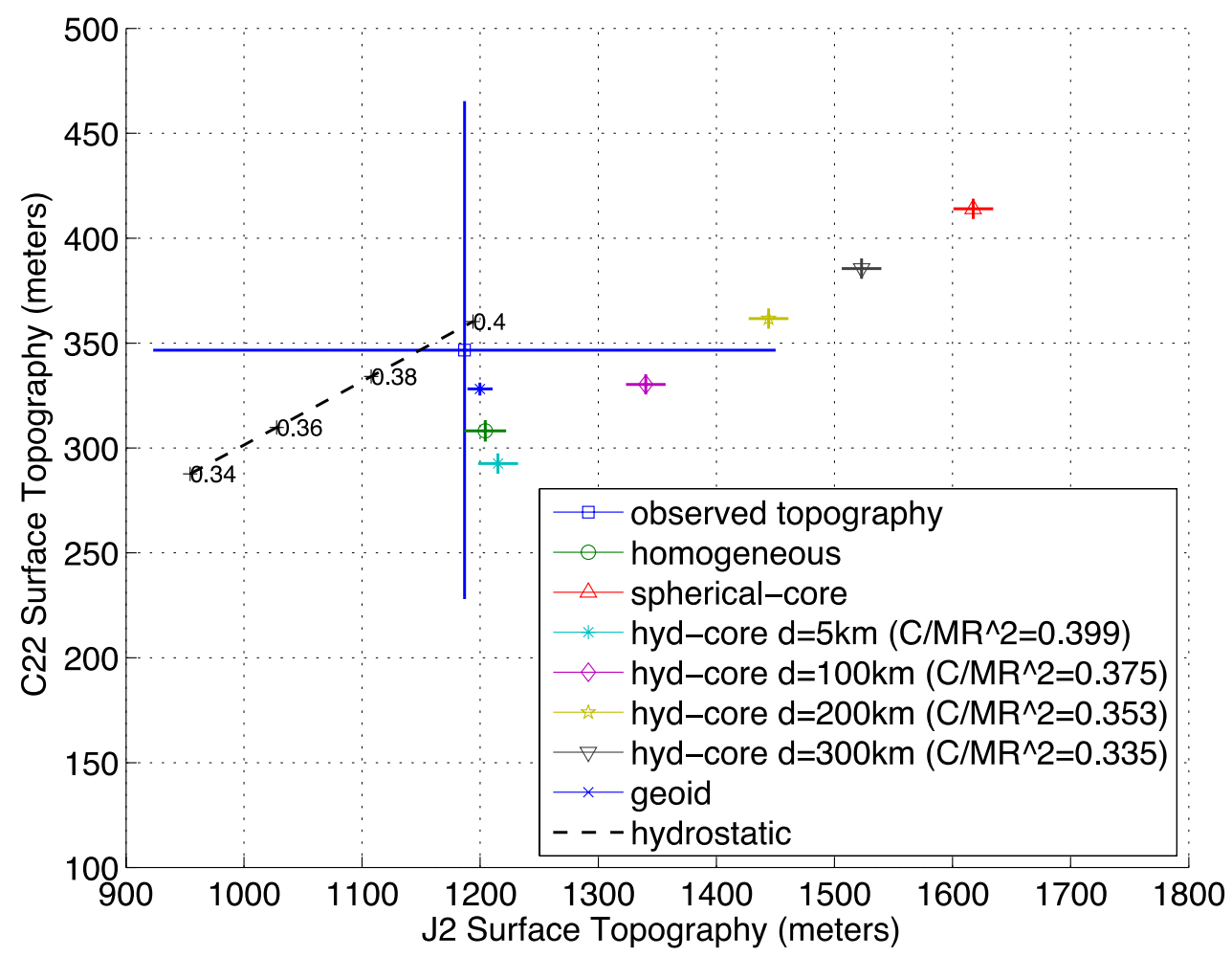

Figure 6 Comparison of observed surface topography (blue square and large crosshairs, representing $1 \sigma$ uncertainties in the shape model) against the topography required to match the measured gravity for the models discussed in Section 3 (each shown 
with crosshairs illustrating the $1 \sigma$ uncertainties propagated from the SOL1 gravity model). For the homogeneous interior case, the shape was determined via equation (10). For the spherical core case, the shape was determined via equation (11), with $H_{l m}^{c}$ set to zero. For the hydrostatic core (hyd-core) cases, the core shape, $\boldsymbol{H}_{l m}^{c}$, was determined using a theory-of-figures approach for a two-layer fluid body (e.g., Tricarico, 2014), assuming various mantle thicknesses (d). Corresponding normalized moments of inertia $\left(C / M R^{2}\right)$ are also given in parentheses. Finally, the geoid was obtained via equation (12). For reference, the surface topography expected for a hydrostatic two-layer body is indicated for various normalized moments of inertia along the dashed black line.

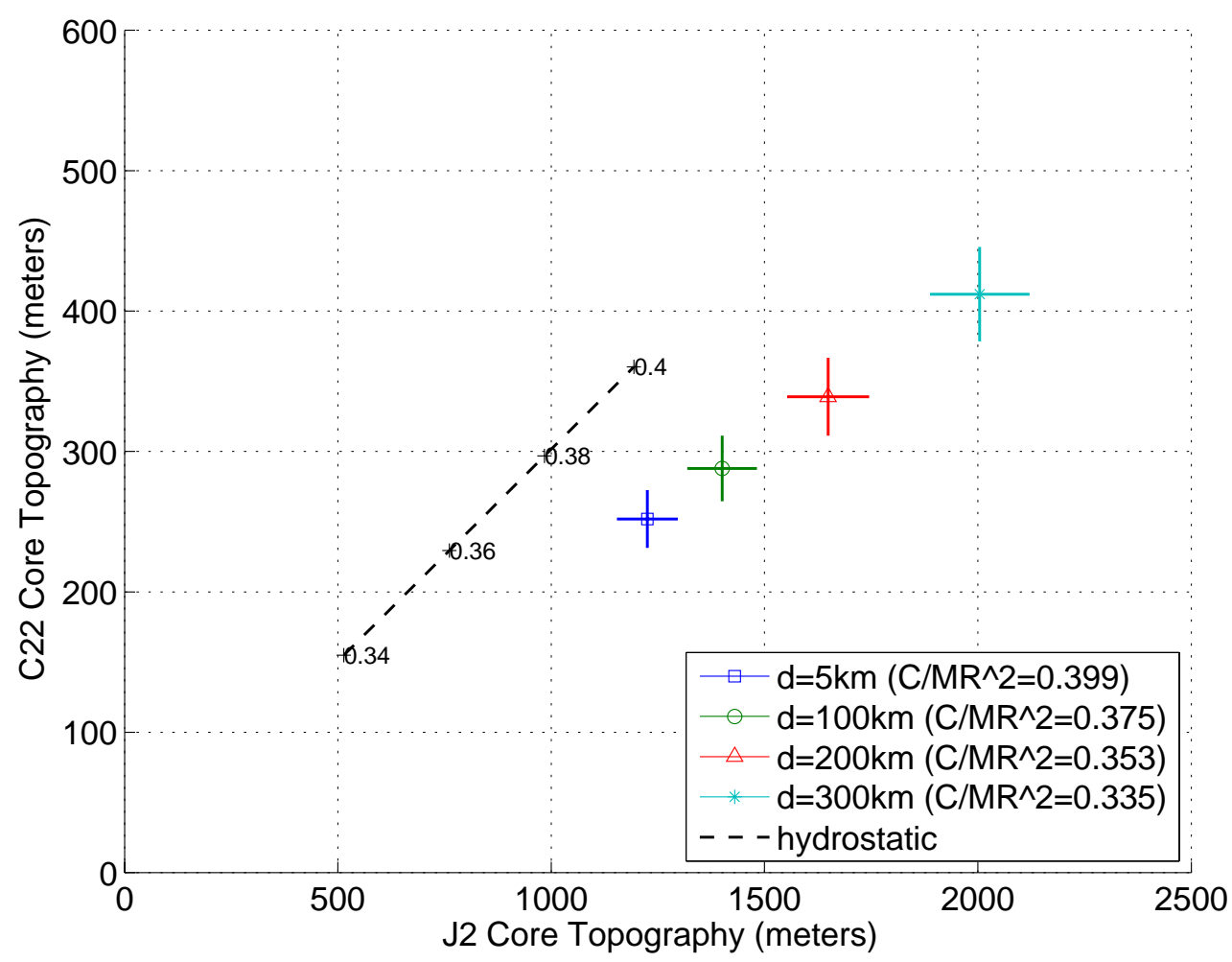

Figure 7 Core topography required to give rise to the observed gravity under the assumption that the degree-2 surface topography conforms to the geoid. The required core topography is computed via equation (11) and depends on the assumed mantle thickness $(d)$; four examples are shown. In each case, the crosshairs illustrate the $1 \sigma$ uncertainties propagated from the SOL1 gravity model. For reference, the core topography expected for a hydrostatic two-layer body is indicated for various normalized moments of inertia along the dashed black line. 


\section{Tables}

Table 1 Main geometrical and orbital characteristics of R1 and R4 gravity fybys.

\begin{tabular}{|l|l|l|l|}
\hline Values at C/A & Unit & R1 & R4 \\
\hline Epoch & $(\mathrm{UTC})$ & $26-\mathrm{NOV}-2005,23: 50$ & $09-\mathrm{MAR}-2013,19: 40$ \\
\hline Altitude & $(\mathrm{km})$ & 502 & 999 \\
\hline Relative velocity & $(\mathrm{km} / \mathrm{s})$ & 7.3 & 9.3 \\
\hline Inclination & $\left({ }^{\circ}\right)$ & 17 & 106 \\
\hline Latitude & $\left({ }^{\circ} \mathrm{N}\right)$ & -10.2 & 18.8 \\
\hline Longitude & $\left({ }^{\circ} \mathrm{E}\right)$ & -91.5 & -176.2 \\
\hline Normal-to-Earth angle & $\left({ }^{\circ}\right)$ & 106 & 117 \\
\hline Sun-Earth-Probe angle & $\left(^{\circ}\right)$ & 113 & 128 \\
\hline
\end{tabular}

Table 2 Expected $\Delta V$ due to Rhea's gravity during R1 and R4. The reference values are: $G M=153.9398 \mathrm{~km}^{3} / \mathrm{s}^{2}, \mathrm{~J}_{2} \times 10^{6}=931, \mathrm{~J}_{3} \times$ $10^{6}=25$.

\begin{tabular}{|l|l|l|l|}
\hline Contribution & Unit & R1 & R4 \\
\hline$\Delta \mathbf{V}(\mathbf{G M})$ & $(\mathrm{m} / \mathrm{s})$ & 16.7 & 9.4 \\
\hline$\Delta \mathbf{V}\left(\mathbf{J}_{2}\right)$ & $(\mathrm{mm} / \mathrm{s})$ & 5.6 & 1.6 \\
\hline$\Delta \mathbf{V}\left(\mathrm{J}_{\mathbf{3}}\right)$ & $(\mathrm{mm} / \mathrm{s})$ & 0.09 & 0.02 \\
\hline
\end{tabular}

Table 3 Estimated values and 1- $\sigma$ formal uncertainties of Rhea's quadrupole gravity unnormalized coefficients for the different approaches described in this paper compared to the results published in (MacKenzie et al., 2008) and (Anderson \& Schubert, 2010). (MacKenzie et al., 2008) did not provide the correlation between $J_{2}$ and $C_{22}$, a zero value was assumed. (Anderson \& Schubert, 2010) solution was obtained by constraining the $J_{2} / C_{22}$ ratio to the hydrostatic value $10 / 3$. A priori values and uncertainties used for SOL1 are also shown in the first column.

\begin{tabular}{|l|l|c|c|c|c|c|}
\hline & Unit & \multicolumn{1}{l|}{ SOL1 (a priori) } & \multicolumn{1}{l|}{ SOL1 } & \multicolumn{1}{l|}{ SOL2 } & $\begin{array}{l}\text { (Mackenzie } \\
\text { et al., 2008) }\end{array}$ & $\begin{array}{l}\text { (Anderson \& } \\
\text { Schubert, 2010) }\end{array}$ \\
\hline $\mathbf{J}_{\mathbf{2}}$ & $\left(\mathrm{x} 10^{6}\right)$ & $930 \pm 600$ & $946.0 \pm 13.9$ & $957.0 \pm 20.3$ & $931.0 \pm 12.0$ & $892.0 \pm 1.6$ \\
\hline $\mathbf{C}_{\mathbf{2 1}}$ & $\left(\mathrm{x} 10^{6}\right)$ & $0 \pm 300$ & $-19.9 \pm 11.0$ & $0 \pm 0.0$ & $0 \pm 0.0$ & $0 \pm 0.0$ \\
\hline $\mathbf{S}_{\mathbf{2 1}}$ & $\left(\mathrm{x} 0^{6}\right)$ & $0 \pm 300$ & $23.5 \pm 21.3$ & $0 \pm 0.0$ & $0 \pm 0.0$ & $0 \pm 0.0$ \\
\hline $\mathbf{C}_{\mathbf{2 2}}$ & $\left(\mathrm{x} 10^{6}\right)$ & $240 \pm 250$ & $242.1 \pm 4.0$ & $227.0 \pm 6.4$ & $237.2 \pm 4.5$ & $267.6 \pm 4.9$ \\
\hline $\mathbf{S}_{\mathbf{2 2}}$ & $\left(\times 10^{6}\right)$ & $0 \pm 250$ & $-15.3 \pm 5.0$ & $-14.9 \pm 5.0$ & $3.8 \pm 3.8$ & $0 \pm 0.0$ \\
\hline $\mathbf{J}_{\mathbf{2}} / \mathbf{C}_{\mathbf{2 2}}$ & & $3.9 \pm 4.8$ & $3.91 \pm 0.10$ & $4.22 \pm 0.19$ & $3.92 \pm 0.09$ & $3.33 \pm 0.0$ \\
\hline corr $\mathbf{J}_{\mathbf{2}}-\mathbf{C}_{\mathbf{2 2}}$ & & 0.0 & -0.34 & -0.58 & $0.0(\mathrm{~N} / \mathrm{A})$ & 1.00 \\
\hline
\end{tabular}

